\title{
Nuevos abonos a partir de excrementos de insecto: el caso del gusano de la harina (Tenebrio molitor)
}

\author{
New fertilizers from insect's excreta: the mealworm (Tenebrio \\ molitor) case
}

Jorge Poveda Arias ${ }^{1}$

\begin{abstract}
Resumen
El gusano de la harina (Tenebrio molitor) es un coleóptero que en la naturaleza representa una importante plaga de productos derivados de cereales. En su cría a nivel industrial con fines alimenticios se han logrado unas cualidades de sus excrementos que los revalorizan como abono de uso en agricultura, al presentar un balance nutricional y un contenido en materia orgánica superior a los abonos orgánicos actuales del mercado. Además, la presencia del polisacárido quitina en estos excrementos los revaloriza aún más para este nuevo uso, ya que es un compuesto con actividad antimicrobiana, capacidad de promoción del crecimiento vegetal, inductor de resistencias y favorecedor de las relaciones simbióticas. Por otro lado, la microbiota asociada a sus intestinos, y que seguramente acompañe sus deposiciones, puede contener importantes microrganismos beneficiosos para las plantas. Este trabajo se centra en una recopilación bibliográfica (review) de todas las investigaciones realizadas hasta el momento sobre el novedoso uso de estos excrementos.
\end{abstract}

Palabras clave: excrementos; Tenebrio molitor; quitina; microbiota; abono.

\begin{abstract}
The mealworm (Tenebrio molitor) is a coleoptera that in nature represents an important pest of cereal products. In their breeding at the industrial level for food purposes, some qualities of their excrements have been achieved that revalue them as a fertilizer for use in agriculture, since they present a nutritional balance and a content in organic matter superior to the current organic fertilizers of the market. In addition, the presence of the polysaccharide chitin in these excrements revalues them even more for this new use, since it is a compound with antimicrobial activity, ability to promote plant growth, inducing resistance and favoring symbiotic relationships. On the other hand, their gut microbiota, that surely accompanies them excreta, may contain important beneficial microorganisms for the plants. This work focuses on a compilation of all the investigations (review) carried out so far on the novel use of these excrements.
\end{abstract}

Key words: excreta; Tenebrio molitor; chitin; microbiota; fertilizer.

\footnotetext{
${ }^{1}$ Biólogo (18.533-CL) e Investigador Predoctoral de Mealfood Europe S.L. Doctorados Industriales (MINECO) (DI-15-07460). Programa de Doctorado en Ingeniería de Biosistemas (RD 99/2011), Universidad de León. jorgepoveda@mealfoodeurope.com
} 


\section{Introducción}

A la vista de los problemas sanitarios y medioambientales asociados a la masiva y descontrolada utilización de compuestos químicos en la agricultura, se hace necesario buscar nuevas alternativas dentro de los compuestos naturales que puedan ofrecernos los mismos o incluso mejores resultados sin efectos secundarios. En este sentido, los insectos tienen la capacidad de alimentarse de materia orgánica de diferente tipo y producir unos deshechos como resultado de su digestión más fácilmente mineralizables por los microorganismos del suelo y accesibles para las plantas (Shepard \& Minshall, 1981; Kagata \& Olgushi, 2012).

En el caso del nitrógeno, los insectos son capaces de acumular cantidades significativas en sus excrementos y en una forma fácilmente asimilable por las plantas, aumentando su crecimiento. Pero estos niveles de nitrógeno dependen directamente de su dieta, tal y como ha podido observarse en saltamontes, polillas de la col (Mamestra brassicae) y abejas (Kagata \& Olgushi, 2012; Fielding et al., 2013; Mishra et al., 2013).

La revisión bibliográfica realizada en este trabajo se ha realizado mediante el uso de bases de datos como Google Scholar o WOS.

\section{El gusano de la harina (Tenebrio molitor)}

Dentro del orden de los coleópteros o escarabajos (suborden Polyphaga), la familia Tenebrionidae (superfamilia Tenebrionoidea) incluye a más de 20.000 especies en todo el mundo, dentro de las cuales pueden encontrarse unas 200 en la Península Ibérica. Son los denominados escarabajos nocturnos del suelo o escarabajos de las tinieblas o de la oscuridad, y es que su nombre científico proviene del latín tenebra (= tiniebla), referente tanto a sus colores negros o pardos profundos, como a sus hábitos de vida (Juan \& Petitpierre, 1991; Chinery, 1997; Barrientos, 2004; Medvedev \& Iwan, 2006; Fernández-Rubio et al., 2014; Escaso et al., 2015).

El escarabajo molinero (Tenebrio molitor), estado adulto del gusano de la harina, es de color castañonegruzco, con pequeños punteados en los élitros y el pronoto y un tamaño de entre 12 y $17 \mathrm{~mm}$ de longitud y 4 $\mathrm{mm}$ de ancho. Tanto las patas como las antenas presentan un color pardo más claro, siendo estas últimas muy cortas y con artejos engrosados. La larva es amarilla, cilíndrica y alargada, llegando a medir de 20 a $30 \mathrm{~mm}$, con pequeñas patas torácicas. Se encuentra extendido por prácticamente todos los lugares del planeta ligados al hombre, alimentándose principalmente de harina u otros productos de origen cereal como pastas, salvado o galletas, aunque también es capaz de consumir papel, cartón, maderas blandas, carne seca, cueros y cortezas de árboles viejos, pudiendo constituir una importante plaga en almacenes (Howard, 1955; Dierl \& Ring, 1995; Chinery, 1997; Chinery, 2001; Moreno \& Álvarez 2005; Triplehorn et al., 2005; Argueta-Reyes \& RamosMeléndez, 2013; Spang, 2013) (Figura 1).
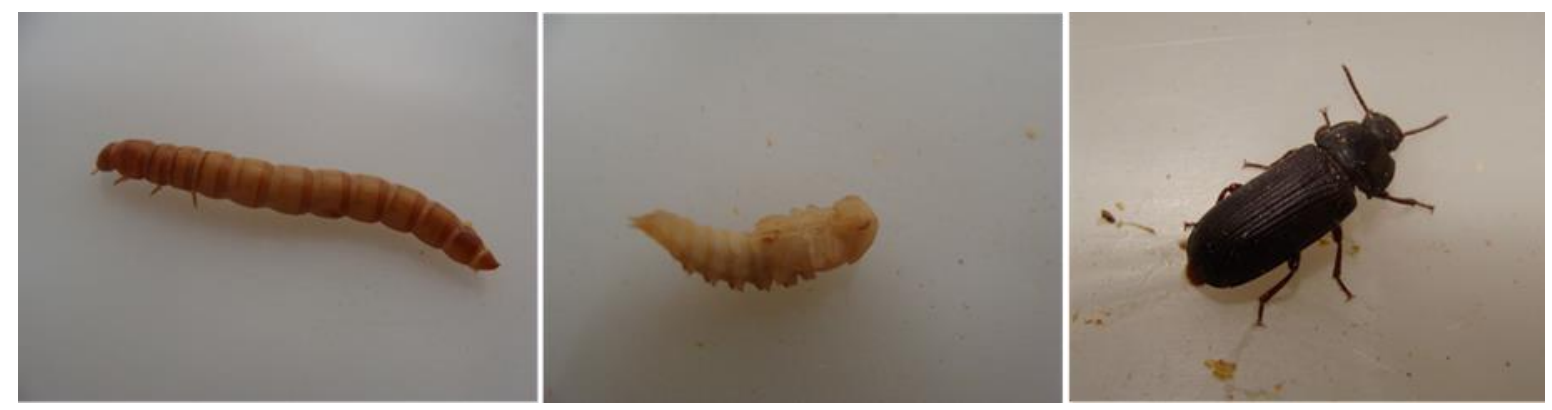

Figura 1: Diferentes estadios de desarrollo de Tenebrio molitor: larva, pupa y escarabajo (de izquierda a derecha). Fotografías: Originales.

En condiciones ambientales, la hembra puede poner de 300 a 600 huevos durante toda su vida, cuya incubación dura un par de semanas, momento en el que surgen las larvas, que se desarrollarán hasta el otoño, pasando por entre 13 y 20 mudas, manteniéndose en estado de hibernación durante el invierno y comenzando su estadio de pupa en primavera, surgiendo los imagos o adultos en pocas semanas. Por lo tanto, en la naturaleza (siempre ligado al hombre) su ciclo de vida es anual, destacando que temperaturas inferiores a $-12^{\circ} \mathrm{C}$ y superiores 
a $41^{\circ} \mathrm{C}$ son letales para la especie (Daggy, 1946; Moreno \& Álvarez 2005; Argueta-Reyes \& Ramos-Meléndez, 2013; Spang, 2013; Park et al., 2014; Escaso et al., 2015).

\section{T. molitor como fuente de abono}

En relación con la agricultura, el gusano de la harina representa una excelente fuente de abono orgánico procedente de sus deposiciones intestinales (Figura 2), cuya composición dependerá de su alimentación. Diferentes análisis químicos realizados en diferentes muestras han dado como resultado balances de NPK de 3,51,5-1,5 (Liu et al., 2002) o de 3,4-1-1,4 (Gao, 2011). En la actualidad, se ha conseguido un balance NPK en dicho abono de 4-4-3, acumulando cantidades significativas de nitrógeno como factor de crecimiento y desarrollo, fósforo como factor de precocidad y potasio como factor de calidad. Además, estos niveles resultan ser mucho más altos y balanceados que en comparación con otros abonos orgánicos utilizados a día de hoy, como son el compost vegetal (1,5-0,8-1), el estiércol vacuno $(0,6-0,4-1)$, porcino $(0,7-0,3-1,6)$, equino $(0,7-0,3-0,8)$, ovino $(0,7-0,5-1,5)$, gallinaza $(1,4-1,6-1,9)$, palomina $(1,8-1,9-1)$, o el humus de lombriz $(2-1,5-1)$, todos ellos con el problema asociado de su alta humedad, de hasta el $80 \%$, lo cual dificulta su estabilidad y almacenaje. Por otro lado, este abono presenta un contenido en materia orgánica muy superior a cualquier otro, de entorno al $80 \%$, mejorando las propiedades físicas (permeabilidad), químicas (complejo de cambio) y biológicas (microorganismos, respiración raíces) del suelo donde es aplicado (Julca-Otiniano et al., 2006; García-Serrano et al., 2010; Poveda, 2015).

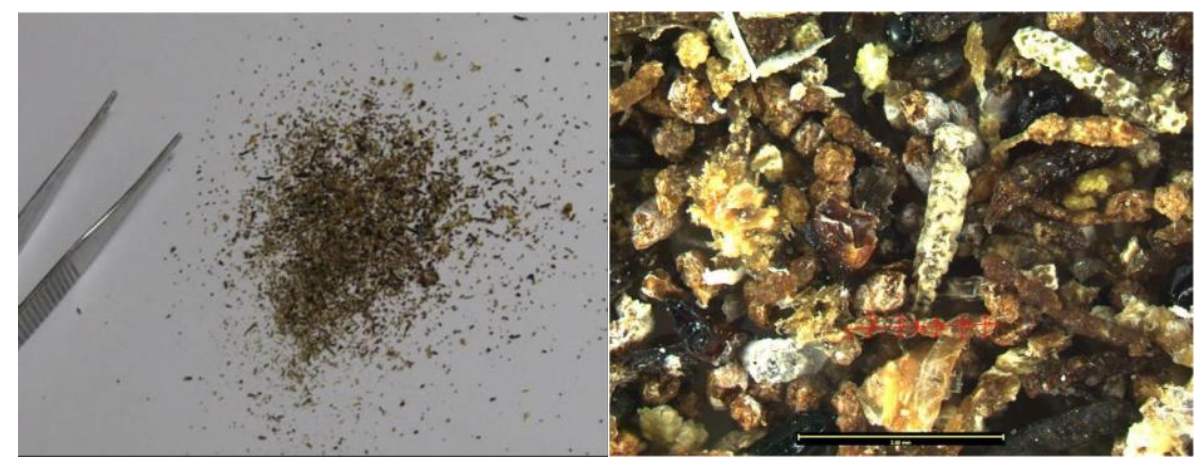

Figura 2: Fotografía de excrementos de T. molitor a tamaño real (izquierda) y a 2,5 aumentos (derecha). La barra representa $2 \mathrm{~mm}$. Extraído de Spang (2013).

En su aplicación directa en cultivos se ha observado que la aplicación de este posible abono provoca en cultivos de judía (Phaseolus vulgaris) un aumento en el peso de la legumbre de hasta un 18\% (Liu et al., 2002), además de un aumento significativo en la productividad de cultivos de colza (Brassica napus) (Luo et al., 2011). Además, la utilización de extractos acuosos a partir de estos excrementos aumenta la germinación de semillas de trigo en un 4\% (Li et al., 2013). Otros estudios se han basado en determinar la seguridad de su uso mediante el análisis de su fitotoxicidad, comprobando como hasta un 2,5\% es totalmente fiable. A partir de ahí, se realizaron estudios en tomate a diferentes concentraciones y en comparación con la aplicación de humus de lombriz. Los resultados obtenidos en dichas investigaciones determinaron como la aplicación del abono procedente de $T$. molitor, con la composición química conseguida por dichos investigadores, a una concentración del 1\% es capaz de provocar un aumento del crecimiento vegetal de un $75 \%$, siendo un $50 \%$ mayor que el que es capaz de provocar el humus de lombriz a la misma concentración, y promover el desarrollo radicular en un 110\%, siendo un $20 \%$ mayor en comparación con el humus. Mientras que al aumentar la concentración hasta un 2,5\% es capaz de provocar un aumento del crecimiento vegetal de un $100 \%$, siendo un $75 \%$ mayor que con el humus de lombriz, y promover el desarrollo radicular en un 200\%, siendo un $110 \%$ mayor en comparación con el humus (Poveda, 2015).

\subsection{La quitina}

Para valorizar aún más la utilización de estas heces como excremento, debemos centrarnos en su proceso de formación. Cuando el alimento pasa a través del intestino medio, el cual carece de cutícula, las células epiteliales segregan una barrera semipermeable denominada membrana peritrófica, que envuelve el alimento y 
está formada por proteínas (peritrofinas) y quitina. Esta membrana tiene como funciones principales encapsular el alimento y dividirlo en distintas porciones, el aislamiento de toxinas y/o patógenos, y la protección del epitelio intestinal. En su interior ocurre la digestión de las macromoléculas con la entrada de enzimas, la cual libera nutrientes al lumen, donde se completa la digestión y absorción de los mismos (Ramsay, 1964; Weiss, 2006; Moreira, 2013). Por lo tanto, cada hez resulta ser un minúsculo gránulo de materia orgánica parcialmente digerida envuelta por una película quitinosa, con lo cual, todos los beneficios de la utilización de la quitina en la agricultura, descritos a continuación, son directamente aplicables a este abono.

La quitina es un polisacárido de $\mathrm{N}$-acetil-glucosaminas unidas entre sí por enlaces $\beta 1 \rightarrow 4$, formando cadenas que se unirán paralelamente para formar microfibrillas (Rinaudo, 2006; Prashanth \& Tharanathan, 2007; Mármol et al., 2013; Younes \& Rinaudo, 2015) (Figura 3). Cuando la quitina es sometida a una reacción de desacetilación capaz de eliminar al menos un $50 \%$ de sus grupos acetilo se convierte a quitosano, y pasa a denominarse quitano si esta desacetilación alcanza el 100\% (Prashanth \& Tharanathan, 2007; Mármol et al., 2013; Younes \& Rinaudo, 2015).

En su utilización en agricultura, la quitina presenta numerosos beneficios como:

- Promotor del crecimiento y desarrollo vegetal, gracias al nitrógeno y los hidratos de carbono que la quitina es capaz de aportar directamente al suelo según se va degradando, a su capacidad para promover el desarrollo de microorganismos beneficiosos, al posible uso del quitosano como anti-transpirante en suelos agrícolas donde la pérdida excesiva de agua es indeseable, o como hidrogeles absorbentes de agua, debido a su alto peso molecular y a su estructura porosa, además, en presencia de quitosano, la planta aumenta la síntesis de las hormonas ácido abscísico (ABA) y ácido jasmónico (JA), ambas relacionadas con la regulación en la apertura de los estomas, aunque pruebas realizadas con mutantes en ambas hormonas siguen respondiendo a la presencia de quitosano con el cierre de los estomas (Bittelli et al., 2001; Sharp, 2013).

- Fitofortificante frente a estreses abióticos, gracias a la actividad del quitosano como antioxidante, dándole la capacidad de secuestrar radicales hidroxilo, peróxido de hidrógeno y anión superóxido, implicados en estreses de carácter abiótico en las plantas. Por lo que se refiere a la contaminación, la quitina y sus derivados son capaces de formar complejos con iones elementales no-nutrientes, como los metales pesados, pudiendo tratar suelos y fuentes de agua contaminados para remediarlos. También es efectivo contra otros contaminantes artificiales de aguas y suelos como pueden ser los tintes, los hidrocarburos, los fertilizantes y los pesticidas químicos (Sharp, 2013).

- Biopesticida antifúngico, ya que el quitosano actúa de igual forma que cuando las enzimas hidrolíticas de las plantas atacan de forma natural a los hongos patógenos y sus células liberan quitosano al romperse su quitina, el cual penetra en sus núcleos interfiriendo con la correcta síntesis de ARN y proteínas (Dutta et al., 2009; Ramírez et al., 2010; Dutta et al., 2012). Además, los grupos amino libres, cargados positivamente en medios ácidos, como el de la rizosfera, interaccionan con los extremos negativos de las macromoléculas expuestas de la pared celular de los hongos, modificando la correcta permeabilidad de su membrana plasmática y, por lo tanto, alterando sus funciones principales (Velásquez, 2008; Ramírez et al., 2010).

- Biopesticida antibacteriano, debido a que el quitosano forma un complejo polielectrolito con la membrana celular externa de bacterias Gram negativas, impidiendo la entrada de nutrientes y la salida de deshechos, provocando la muerte bacteriana, mientras que en las bacterias Gram positivas lo que ocurriría sería la entrada del quitosano por los poros de la membrana hasta el núcleo, impidiendo la síntesis de ARN y proteínas (Velásquez, 2008).

- Biopesticida nematicida, debido a que la presencia de quitina en el suelo favorece el desarrollo de microorganismos quitinolíticos que parasitan los huevos de los nematodos y destruyen la quitina presente en la cutícula de los juveniles, y a que la degradación de la misma produce como residuo amoniaco gaseoso que, al acumularse en determinados niveles, tiene efecto directo nematicida (Velásquez, 2008; Ramírez et al., 2010; Sharp, 2013). 
- Inductor de resistencia, gracias a que el quitosano es percibido por la planta como la presencia de un hongo patógeno o un insecto fitófago, por lo que pre-activa sus defensas vegetales frente a ese tipo de peligros (Kaku et al., 2006; Velásquez, 2008; Enríquez-Guevara et al., 2010; Sharp, 2013).

- Favorecedor de relaciones simbióticas, debido a que la quitina favorece el crecimiento y desarrollo de microorganismos antagonistas de fitopatógenos, como Trichoderma o Beauveria bassiana, mejora la capacidad de rhizobacterias para entrar en simbiosis con leguminosas y de los hongos micorrícicos, al promover su crecimiento micelar (Ramírez et al., 2010; Sharp, 2013).

\subsection{Su microbiota}

Es ampliamente sabido que los insectos atesoran una variadísima microbiota intestinal muy beneficiosa para ellos. Dentro de esta microbiota, T. molitor presenta una serie de bacterias que pueden acompañar a sus deposiciones intestinales y que son beneficiosas para la agricultura. Dentro de ellas podemos incluir (Jung et al., 2014; Wang \& Zhang, 2015; Li et al., 2016; Stoops et al., 2016):

- Paenibacillus sp. es un género de bacterias con un gran interés en agricultura, ya que se incluyen dentro de las denominadas como bacterias promotoras del crecimiento vegetal (PGPRs), al mejorar el aporte de nitrógeno a la planta, la absorción de potasio, calcio y magnesio, la solubilización de fosfatos, la producción de sideróforos y de auxinas, y la tolerancia frente a estreses abióticos por metales pesados, sequía y salinidad; además, presenta actividad antifúngica contra hongos fitopatógenos como Collecotrichum goleosporoides, Fusarium oxysporum, Botrytis cinerea, Phytophthora capsici o Rhizoctonia solani, bacterias como Pectobacterium caratovorum, y nematodos como Meoidogyne incognita; e incluso es capaz de degradar pesticidas organofosforados presentes como residuos en los suelos (Zhang et al., 2013; Romeh \& Hendawi, 2014; Silva et al., 2015; Seo et al., 2016; Xie et al., 2016).

- Bacillus sp., género de bacterias PGPRs cuya representante más conocida es B. thuringiensis, debido a su capacidad para sintetizar una proteína insecticida muy eficaz. Pero existen otras especies capaces de estimular la germinación y el crecimiento de las plántulas, fijar el nitrógeno atmosférico, producir sideróforos, solubilizar fosfatos, y producir auxinas que favorecen la formación de raíces por las plantas, además es capaz de inducir en la planta una resistencia sistémica frente al ataque de plagas y patógenos, e incluso tiene acción directa antifúngica contra Fusarium graminearum (Figura 3), F. incarnatum, Collecotrichum acutatum y $C$. goleosporoides, antibacteriana contra Ralstonia solanacearum, nematicida contra Meloidogyne incognita y supresor del protista causante de la hernia de la col, Plasmodiophora brassicae (Peng et al., 2013; Zhao et al., 2014; Ab Aziz et al., 2015; Han \& Kim, 2015; Kaur et al., 2015; Widnyana \& Javandira, 2016).

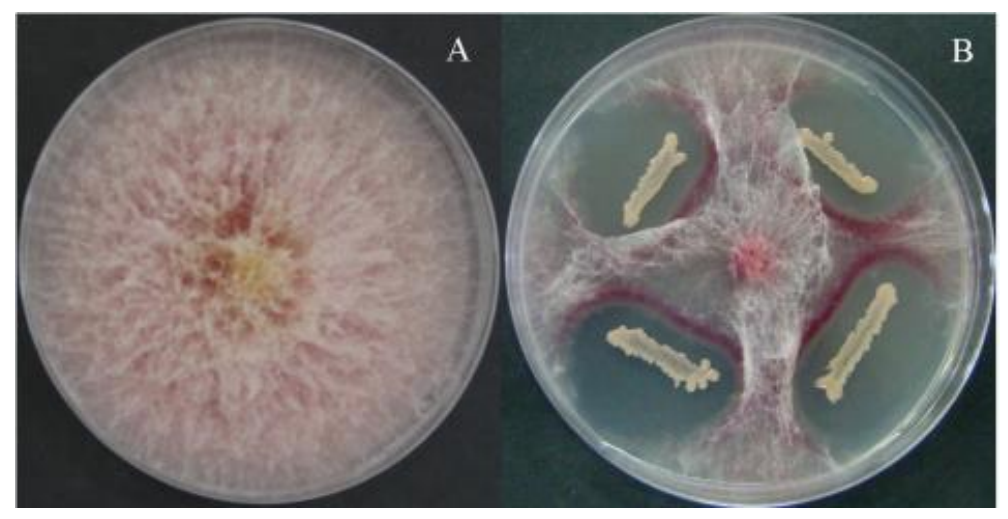

Figura 3: Efecto antifúngico de Bacillus subtilis sobre Fusarium graminearum, tras 5 días de incubación. Extraído de Zhao et al. (2014).

- Rhizobium sp., es el género de bacterias PGPRs más estudiado (Figura 4), son capaces de fijar el nitrógeno atmosférico, solubilizar fosfatos, producir auxinas y sideróforos, además, una vez que han conformado su relación simbiótica con las leguminosas a las que les aportan estos beneficios, son capaces de liberar 
cianuro de hidrógeno en forma gaseosa al suelo, impidiendo el crecimiento cercano de malas hierbas, e incluso son capaces de inducir en la planta una resistencia sistémica frente a diferentes patógenos (Baba et al., 2015; Singh et al., 2015). Por otro lado, son capaces de inducir en las plantas tolerancia frente a estrés salino (Elsheikh, 2015) y sequía (Rasaei et al., 2013; Beshir et al., 2015).

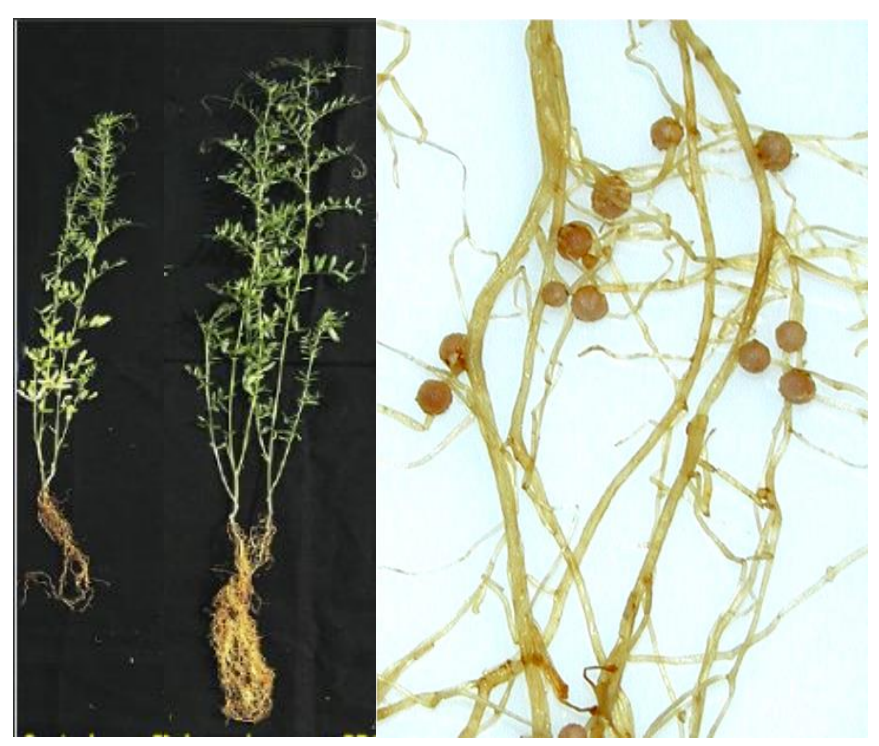

Figura 4: Efecto de promoción del crecimiento de Rhizobium leguminosarum en lenteja (derecha), frente al control (izquierda); y vista de los nódulos radiculares que forma Rhizobium japonicum en soja. Extraído de Mishra et al. (2012) y Ferguson (2013).

El papel de la microbiota presente en los excrementos de T. molitor como microorganismos inhibidores del crecimiento y desarrollo de patógenos ha dado resultados prometedores en su enfrentamiento contra fitopatógenos como Ralstonia solanacearum, Pectobacterium carotovorum, Phytophthora cactorum, Fusarium oxysporum $\mathrm{f}$. sp. melonis y Rhizoctonia solani (Poveda, 2017).

\section{Conclusiones}

Los excrementos de los insectos representan un buen aporte de nutrientes para las plantas. En el caso de Tenebrio molitor, sus excrementos presentan un balance nutricional y un contenido en materia orgánica muy bueno para su uso como abono en agricultura. Además, los excrementos de T. molitor presentan quitina, compuesto químico con cualidades bioestimulantes del crecimiento vegetal, fitofortificantes y biopesticidas de organismos fitopatógenos. Por otro lado, la microbiota asociada a estos excrementos puede presentar microorganismos muy valiosos para su uso en agricultura.

\section{Referencias Bibliográficas}

Ab Aziz, Z. F., Saud, H. M., Kundat, F. R., Jiwan, M., \& Wong, S. K. 2015. Rhizobacterium Bacillus cereus induces root formation of pepper (Piper nigrum L.) stem cuttings. Research in Biotechnology, 6(2).

Argueta-Reyes, L. \& Ramos-Meléndez, G.K. 2013. Contenido de Proteína, Grasa, Calcio, Fósforo en larvas del escarabajo molinero (Coleoptera: Tenebrionidae: Tenebrio molitor L.) alimentadas con diferentes sustratos y fuentes de agua; para ser utilizadas como alimentación de animales silvestres. Tesis Doctoral. Universidad de El Salvador.

Baba, Z. A., Aziz, M. A., Sheikh, T. A., Sheikh, F. A., Bhat, Z. A., Khan, S., \& Hamid, B. 2015. Studies on soil health and plant growth promoting potential of Rhizobium isolates. Emirates Journal of Food and Agriculture, 27(5), 423. 
Barrientos, J.A. 2004. Curso práctico de entomología (Vol. 41). Univ. Autónoma de Barcelona.

Beshir, H. M., Walley, F. L., Bueckert, R., \& Tar'an, B. 2015. Response of snap bean cultivars to Rhizobium inoculation under dryland agriculture in Ethiopia. Agronomy, 5(3), 291-308.

Bittelli. M., Flury, M., Campbell, G.S., Nichols, E.J. 2001. Reduction of transpiration through foliar application of chitosan. Agricultural and Forest Meteorology, 107: 167-175.

Chinery, M. 1997. Guía de campo de los insectos de España y de Europa. Editorial Omega.

Chinery, M. 2001. Guía de los insectos de Europa. Editorial Omega.

Daggy, T. 1946. Notes on the ecology and taxonomy of certain pupae of the family Tenebrionidae (Coleoptera). Proceedings of the Indiana Academy of Science, 56: 253-260.

Dierl, W. \& Ring, W. 1995. Insectos de España y de Europa: identificación, hábitat, biología. Editorial Omega.

Dutta, J., Tripathi, S., Dutta, P.K. 2012. Progress in antimicrobial activities of chitin, chitosan and its oligosaccharides: a systematic study needs for food applications. Food Science and Technology International, 18: 3-34.

Dutta, P.K., Tripathi, S., Mehrotra, G.K., Dutta, J. 2009. Perspectives for chitosan based antimicrobial films in food applications. Food Chemistry, 114: 1173-1182.

Elsheikh, E. A. 2015. Response of legume-Rhizobium symbiosis to salinity in the Sudan: a review. Journal of Agricultural Science, 6(2), 142-156.

Enríquez-Guevara, E.A., Aispuro-Hernández, E., Vargas-Arispuro, I., Martínez-Téllez, M.Á. 2010. Oligosacarinas Derivadas de Pared Celular: Actividad Biológica y Participación en la Respuesta de Defensa de Plantas. Revista Mexicana de Fitopatología, 28:144-155.

Escaso, F., Narváez, I., Planelló, M.R., Rueda, M.J., Herrero, O. 2015. Manual de Entomología Aplicada. Sanz y Torres-UNED.

Ferguson, B. J. 2013. The development and regulation of soybean nodules. INTECH Open Access Publisher.

Fernández Rubio, F., Moreno Fernández-Caparrós, L., Soriano, Ó. 2014. Artrópodos en medicina y veterinaria. Ministerio de Defensa de España.

Fielding, D. J., Trainor, E., \& Zhang, M. 2013. Diet influences rates of carbon and nitrogen mineralization from decomposing grasshopper frass and cadavers. Biology and Fertility of Soils, 49(5), 537-544.

Gao Y (2011). 黄粉虫粪营养成分的测定与分析 (Measurement and analysis Tenebrio faecal nutrients). 黑龙 江畜牧兽医: 下半月, (3).

García-Serrano, P., Ruano, S., Marotta, J., \& Nogales, M. 2010. Guía práctica de la fertilización racional de los cultivos en España. Parte I. Gobierno de España. Ministerio del Medio Ambiente y Medio Rural y Marino. España.

Goswami, D., Parmar, S., Vaghela, H., Dhandhukia, P., \& Thakker, J. N. 2015. Describing Paenibacillus mucilaginosus strain $\mathrm{N} 3$ as an efficient plant growth promoting rhizobacteria (PGPR). Cogent Food \& Agriculture, 1(1), 1000714.

Han, J. H., \& Kim, J. O. 2015. D-6: Biological Control of Anthracnose (Colletotrichum acutatum and C. gloeosporioides) on Pepper by Use of Bacillus spp. 균학회소식, 27(1), 116-116.

Howard, R.S. (955. The biology of the grain beetle Tenebrio molitor with particular reference to its behavior. Ecology, 36: 262-269.

Juan, C. \& Petitpierre, E. 1991. Evolution of genome size in darkling beetles (Tenebrionidae, Coleoptera). Genome, 34: 169-173.

Julca-Otiniano, A., Meneses-Florián, L., Blas-Sevillano, R., Bello-Amez, S. 2006. La materia orgánica, importancia y experiencia de su uso en la agricultura. Idesia, 24: 49-61. 
Jung, J., Heo, A., Park, Y.W., Kim, Y.J., Koh, H., Park, W. 2014. Gut microbiota of Tenebrio molitor and their response to environmental change. J. Microbiol. Biotechnol, 24: 888-897.

Kagata, H., \& Ohgushi, T. 2012. Positive and negative impacts of insect frass quality on soil nitrogen availability and plant growth. Population Ecology, 54(1), 75-82.

Kaku, H., Nishizawa, Y., Ishii-Minami, N., Akimoto-Tomiyama, C., Dohmae, N., Takio, K., Shibuya, N. 2006. Plant cells recognize chitin fragments for defense signaling through a plasma membrane receptor. Proceedings of the National Academy of Sciences, 103: 11086-11091.

Kaur, H., Kaur, H., \& Rishi, P. 2015. Bare-root-dip treatment of tomato seedlings with neem and Bacillus thuringiensis for the management of Meloidogyne incognita. History, 3(10), 6-17.

Li, L., Xie, B., Dong, C., Wang, M., Liu, H. 2016. Can closed artificial ecosystem have an impact on insect microbial community? A case study of yellow mealworm (Tenebrio molitor L.). Ecological Engineering, 86: 183-189.

Li, L., Zhao, Z., Liu, H. 2013. Feasibility of feeding yellow mealworm (Tenebrio molitor L.) in bioregenerative life support systems as a source of animal protein for humans. Acta Astronautica, 92: 103-109.

Liu, H., Yang, Z., Tan, D., \& Wu, Z. 2002. Study on the fertilizer efficiency of the frass of Tenebrio molitor L. Journal of Quanzhou Normal College (Natural Science), 21(4), 68-71.

Luo, H. Y., Wang, H., \& Wang, Q. 2011. Effects of Different Application Amount of Yellow Mealworm Excrement on Growth and Quality of Rape. Shandong Agricultural Sciences, 8, 026.

Mármol, Z., Páez, G., Rincón, M., Araujo, K., Aiello, C., Chandler, C., \& Gutiérrez, E. 2013. Quitina y Quitosano polímeros amigables. Una revisión de sus aplicaciones/Chitin and Chitosan friendly polymer. A review of their applications. Revista Tecnocientífica URU, (1), 53-58.

Medvedev, G.S. \& Iwan, D. 2006. Notes on the taxonomy of tenebrionid beetles (Coleoptera: Tenebrionidae). Annales Zoologici, 56: 613-621.

Mishra, A., Afik, O., Cabrera, M. L., Delaplane, K. S., \& Mowrer, J. E. 2013. Inorganic nitrogen derived from foraging honey bees could have adaptive benefits for the plants they visit. PloS one, 8(7), e70591.

Mishra, P. K., Bisht, S. C., Mishra, S., Selvakumar, G., Bisht, J. K., \& Gupta, H. S. 2012. Coinoculation of Rhizobium leguminosarum-PR1 with a cold tolerant Pseudomonas sp. improves iron acquisition, nutrient uptake and growth of field pea (Pisum sativum L.). Journal of plant nutrition, 35(2), 243-256.

Moreira, N.R. 2013. Fisiologia molecular intestinal de Tenebrio molitor. Doctoral dissertation, Universidade de São Paulo.

Moreno, A.A. \& Alvarez, C.S. 2005. Entomología agraria: los parásitos animales de las plantas cultivadas. Diputación Provincial de Soria.

Park, J.B., Kim, S.H., Han, Y.S., Kim, N.J. 2014. Developmental characteristics of Tenebrio molitor larvae (Coleoptera: Tenebrionidae) in different instars. International Journal of Industrial Entomology, 28: 5-9.

Peng, G., Pageau, D., Strelkov, S. E., Lahlali, R., Hwang, S. F., Hynes, R. K., \& Falk, C. K. 2013. Assessment of crop rotation, cultivar resistance and Bacillus subtilis biofungicide for control of clubroot on canola. Acta Hort, 1005, 591-598.

Poveda, J. 2015. Mealfrass: Utilización de excrementos del gusano de la harina (Tenebrio molitor) como abono orgánico con capacidad bioestimulante. XXXI Encuentro de Jóvenes Investigadores. Instituto de Investigaciones Científicas y Ecológicas.

Poveda, J. 2017. Mealfrass III: Utilización de excrementos del gusano de la harina (Tenebrio molitor) como abono orgánico con capacidad biopesticida. XXXIII Encuentro de Jóvenes Investigadores. Instituto de Investigaciones Científicas y Ecológicas.

Prashanth, K.H. \& Tharanathan, R.N. 2007. Chitin/chitosan: modifications and their unlimited application potential - an overview. Trends in Food Science \& Technology, 18: 117-131. 
Ramírez, M.A., Rodríguez, A.T., Alfonso, L., Peniche, C. 2010. Chitin and its derivatives as biopolymers with potential agricultural applications. Biotecnología Aplicada, 27: 270-276.

Ramsay, J.A. 1964. The rectal complex of the mealworm Tenebrio molitor, L. (Coleoptera, Tenebrionidae). Philosophical Transactions of the Royal Society of London B: Biological Sciences, 248: 279-314.

Rasaei, B., Ghobadi, M. E., Ghobadi, M., \& Najaphy, A. 2013. Reducing effects of drought stress by application of humic acid, mycorrhiza and Rhizobium on chickpea. International Journal of Agriculture and Crop Sciences, 5(16), 1775.

Rinaudo M (2006). Chitin and chitosan: properties and applications. Progress in Polymer Science, 31: 603-632.

Romeh, A. A., \& Hendawi, M. Y. 2014. Bioremediation of certain organophosphorus pesticides by two biofertilizers, Paenibacillus (Bacillus) polymyxa (Prazmowski) and Azospirillum lipoferum (Beijerinck). Journal of Agricultural Science and Technology, 16(2), 265-276.

Seo, D. J., Lee, Y. S., Kim, K. Y., \& Jung, W. J. 2016. Antifungal activity of chitinase obtained from Paenibacillus ehimensis MA2012 against conidial of Collectotrichum gloeosporioides in vitro. Microbial Pathogenesis, 96, 10-14.

Sharp, R.G. 2013. A review of the applications of chitin and its derivatives in agriculture to modify plantmicrobial interactions and improve crop yields. Agronomy, 3: 757-793.

Shepard, R. B., \& Minshall, G. W. 1981. Nutritional value of lotic insect feces compared with allochthonous materials. Archiv fur Hydrobiologie.

Silva, V. N. D., Silva, L. E., da Silva, A. J., \& Macedo, G. R. D. 2015. Biofertilizers and performance of Paenibacillus in the absorption of macronutrients by cowpea bean and soil fertility. Revista Brasileira de Engenharia Agrícola e Ambiental, 19(12), 1136-1142.

Singh, A. D. R. K., Kumar, S., \& Kumar, S. 2015. An effective and beneficial plant growth promoting soil bacterium "Rhizobium": a review. Annals of Plant Sciences, 4(01), 933-942.

Spang, B. 2013. Insects as food: Assessing the food conversion efficiency of the mealworm (Tenebrio molitor). Doctoral dissertation, The Evergreen State College.

Stoops, J., Crauwels, S., Waud, M., Claes, J., Lievens, B., Van Campenhout, L. 2016. Microbial community assessment of mealworm larvae (Tenebrio molitor) and grasshoppers (Locusta migratoria migratorioides) sold for human consumption. Food Microbiology, 53: 122-127.

Triplehorn, C.A.J., Borror, N.F., Triplehorn, D.J.C.A., Johnson, N.F. 2005. Introduction to the study of insects. Thomson-Brooks.

Velásquez, C.L. 2008. Algunas potencialidades de la quitina y el quitosano para usos relacionados con la agricultura en Latinoamérica. Revista UDO Agrícola, 8: 1-22.

Waewthongrak, W., Pisuchpen, S., \& Leelasuphakul, W. 2015. Effect of Bacillus subtilis and chitosan applications on green mold (Penicilium digitatum Sacc.) decay in citrus fruit. Postharvest Biology and Technology, 99, 44-49.

Wang, Y. \& Zhang, Y. 2015. Investigation of Gut-Associated Bacteria in Tenebrio molitor (Coleoptera: Tenebrionidae) Larvae Using Culture-Dependent and DGGE Methods. Annals of the Entomological Society of America, 108: 941-949.

Widnyana, I. K., \& Javandira, C. 2016. Activities Pseudomonas spp. and Bacillus sp. to Stimulate Germination and Seedling Growth of Tomato Plants. Agriculture and Agricultural Science Procedia, 9, 419-423.

Xie, J., Shi, H., Du, Z., Wang, T., Liu, X., \& Chen, S. 2016. Comparative genomic and functional analysis reveal conservation of plant growth promoting traits in Paenibacillus polymyxa and its closely related species. Scientific Reports, 6 .

Younes, I., \& Rinaudo, M. 2015. Chitin and chitosan preparation from marine sources. Structure, properties and applications. Marine drugs, 13(3), 1133-1174. 
Zhang, M.I., Tan, T., Yuan, H., Rui, C. 2003. Insecticidal and fungicidal activities of chitosan and oligochitosan. Journal of Bioactive and Compatible Polymers, 18: 391-400.

Zhang, A. M., Zhao, G. Y., Gao, T. G., Wang, W., Li, J., Zhang, S. F., \& Zhu, B. C. 2013. Solubilization of insoluble potassium and phosphate by Paenibacillus kribensis CX-7: A soil microorganism with biological control potential. African Journal of Microbiology Research, 7(1), 41-47.

Zhao, Y., Selvaraj, J. N., Xing, F., Zhou, L., Wang, Y., Song, H., \& Liu, Y. 2014. Antagonistic action of Bacillus subtilis strain SG6 on Fusarium graminearum. PLoS One, 9(3), e92486. 